\title{
LA MÉMOIRE DES PHRASES
}

(MÉMOIRE DES IDÉES)

Bien que cette étude soit la continuation logique et le développement de celle que nous venons d'écrire sur la mémoire verbale des mots isolés, bien que nous ayons employé pour ces deux recherches les mêmes méthodes, nous croyons cependant utile de traiter les deux questions dans deux articles distincts. Voici pourquoi.

La mémoire verbale des mots isolés a déjà été étudiée par de nombreux observateurs; ce n'est pas un sujet entièrement nouveau et inexploré; d'autres formes de la mémoire qui se rapprochent beaucoup de la mémoire verbale, ont été prises aussi cette année même comme objet d'expériences par M. Münsterberg et plusieurs de ses élèves, M. Bigham,

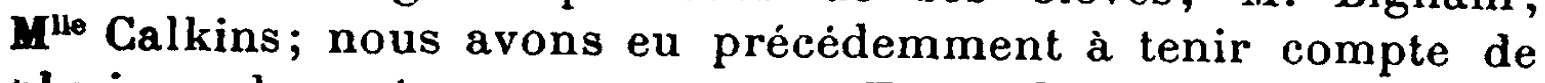
plusieurs de ces travaux nouveaux. Tout cela forme un ensemble de recherches, qui avancent rapidement, sur ce qu'on peut appeler la mémoire des sensations; c'est là le caractère principal de ces recherches; elles ont trait à la mémoire d'éléments de conscience relativement simples; les carrés de couleur dont on cherche à retenir l'assemblage ou la nuance exacte dans la mémoire sont des éléments presque entièrement 'sensoriels; les chiffres qu'on cherche à retenir donnent principalement une sensation pour l'œil et pour l'oreille, et l'idée de pluralité d'objets qu'íls éveillent est assez vague; pour les mots, la suggestion d'idées est plus nette et mieux définie; et certainement ces suggestions d'idées que les mots provoquent doivent exercer une influence sur la mémoire des mots ; mais dans la forme où 
l'on fait les expériences de ce genre, on ne peut guère étudier séparément ce qu'on peut appeler la mémoire des idées, et l'isoler de la mémoire des mots; pour toutes ces raisons, on doit conclure que les expériences de mémoire ont porté jusqu'ici presque uniquement sur la mémoire des sensations.

Arec nos présentes études sur la mémoire des phrases, nous abordons bien réellement la mémoire des idées.

C'est là ce qui donne un très vif intérêt à nos nouvelles recherches, et nous entendons les séparer des précédentes pour bien montrer que nous entrons ici dans un domaine entièrement inexploré.

Peu d'explications seront nécessaires pour faire comprendre la méthode que nous avons suivie; c'est la méthode collective que nous avons déjà employée pour la mémoire verbale des mots isolés. Les expériences ont été faites dans quatre écoles primaires élémentaires de Paris ; elles ont porté sur 510 élèves; un morceau de prose d'une longueur variable était lu par le professeur aux élèves en notre présence ; les élèves étaient avertis d'avance qu'après avoir entendu le morceau ils auraient à l'écrire de mémoire' ${ }^{1}$; aussitôt après la lecture, ils commençaient à écrire; et aussitôt après qu'ils avaient fini leur rédaction, les copies étaient ramassées et nous étaient remises. L'expérience collective se faisait très rapidement, et causait aux élèves un dérangement très court; en une demi-heure au plus tout était terminé ; ce qui était beaucoup plus long, c'était notre propre travail, consistant dans l'examen des copies, la numération des erreurs, le calcul des tables et le dessin des graphiques. i Nos expériences ont porté sur des phrases ou des paragraphes

(1) Cette orientation particulière de l'attention est fort importante a connaitre, car elle exerce une influence très grande, plus grande qu'on ne le croit en général, sur les effets des expériences. Deux travaux que nous avons analysés dans cette Annéc, celui de Münsterberg sur le Pouvoir moteur des idées, et celui de Miss Calkins sur l'Association des idées, le démontrent. En se reportant à ces articles, on verra que lorsqu'on présente pour la seconde fois le même objet à une personne (expérience de llünsterberg) le mouvement de ses yeux indique que l'intérêt qu'elle prend à cet objet et son attention décroissent, tout simplement parce que la personne qui sert de sujet obéit à sa tendance naturelle à trouver monotonc et ennuyeux ce qui se répète; si au contraire cette personne sait qu'elle doit retenir les objets qu'on lui prèsente, et s'efforce de le faire comme dans les expériences de lliss Calkins, la répétition d'une méme impression en assure la conservation dans la mèmoire, el doit éveiller un tout autre genre d'état de conscience que l'ennui. C'est ainsi qu'avec deux attitudes d'esprit différentes, un mème stimulus extérieur produit des effets inverses. 
dictés qui se composaient d'un nombre variable de mots. Nous donnons ci-après le nombre de mots des dictées, avec le nombre d'élèves sur lesquels les expériences ont èté faites :

$$
\begin{aligned}
& \text { Dictée de } 11 \text { mots dans une classe de } 306 \text { élèves. } \\
& \text { - } 14 \text { mots . . . . . 30з̆ élèves. } \\
& \text { - } 20 \text { mots . . . . . . } 303 \text { élèves. } \\
& \text { - } 38 \text { mots . . . . . . } 64 \text { élèves. } \\
& \text { - } 60 \text { mots . . . . . . . } 77 \text { élèves. } \\
& \text { - } 74 \text { mots . . . . . . } 36 \text { élèves. } \\
& \text { - } 86 \text { mots . . . . . } 36 \text { élèves. }
\end{aligned}
$$

Nous ne tiendrons compte que des expériences dont nous avons entièrement calculé les résultats ; nous avons fait des expériences collectives beaucoup plus nombreuses; mais nous ne les avons pas calculées entièrement, parce que ces calculs nous auraient coûté plusieurs mois de travail; nous nous sommes contentés de parcourir plusieurs centaines de ces copies, et de nous assurer par un rapide coup d'œil que l'ensemble des résultats était conforme à ceux que nous avions calculés.

Voici la série de dictées que nous avons utilisées; nous les transcrivons entièrement, parce que le lecteur a besoin de les connaitre pour comprendre nos analyṣes ultérieures.

Texte de 20 mots et de 8 groupes de mots.

Le petit Emile | a obtenu | de sa mère | un joli | cheval mécanique | en récompense | de sa bonne conduite | à l'école.

Texte de 14 mots et de 8 groupes.

Son ami | s'amusera | avec lui | le jeudi | et le dimanche | une fois | son devoir | terminé.

Texte de 11 mots et de 5 groupes.

Si le cheval | se brise, | un mécanicien | le réparera | de suite.

Texte de 38 mots et de 17 groupes.

Imprudence d'enfants. | Dimanche | plusieursenfants | s'amusaient | à faire marcher | une machine à mortier ; | l'un d'eux, | Victor Antiquel, | eut sa main | gauche | écrasée | dans l'engrenage. | Il a reçu | les premiers soins.| dans une pharmacie | d'où il a èté porté | cheq̣ ses parents.

Texte de 60 mots et de 19 groupes.

Une vieille paysanne | âgée de 64 ans, | la veuve Mouillet, | 


\section{A. BINET ET V. hENR. - LA MÉNOIRE DES PHRASES}

qui habitait une petite maison | sur la route déserte | des Recolets, | avait conduit I son troupeau | dans les champs. I Pendant qu'elle faisait de l'herbe pour ses animaux | une vipère | cachée derrière les fagots | s'élança sur elle | et la mordit | à plusieurs reprises | au poignet. | La pauvre | femme | en est morte.

Texte de 74 mots et de 20 groupes.

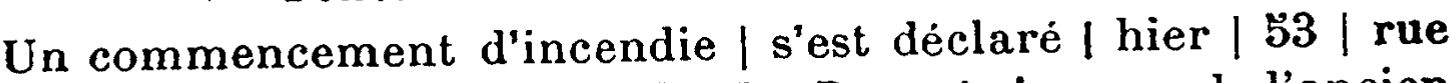
Jacob | dans l'appartement | de $\mathbf{M}^{\mathbf{m}^{\mathrm{e}}}$ Bequet, | veuve de l'ancien conseiller d'État. | Le feu a pris naissance | dans le salon | par suite de l'imprudence | de $M^{\text {me }}$ Bequet |qui en tenant une bougie allumée | l'avait approchée | trop près des tentures | devant les murs. | Les pompiers | avertis à temps | ont pu éteindre | rapidement | ce commencement d'incendie, | mais des tapis persans | d'un grand prix | ont été dévorés par le feu.

Texte de 86 mots et de 24: groupes.

Un fait bien curieux | vient de se passer / en mer : | un vapeur, | qui porte le nom | d'Adèle, | se trouvait | il y a quelques jours | devant la côte | de Penmarch, | où les écueils sont nombreux ; | ce bâtiment | ayantépuisé | sa provision | de charbon, | ne voyant aucun autre navire | capable de le remorquer | ne savait comment se tirer | de ce mauvais pas; | le capitaine Bernard | s'est décidé | à chauffer | sa machine | en faisant brûler | deux canots, | les mâts de rechange | et le bois de soixante barriques de vin.

\section{II}

La première question que nous avons à examiner est celle de la quantité de souvenirs que les enfants retiennent après une seule audition. C'est une question avec laquelle nous sommes déjả familiarisés, car nous l'avons posée pour la mémoire des mots.

$\checkmark$ Une première difficulté se présente dans la mesure de la mémoire verbale des phrases. Les phrases sont formées de nots dont chacun n'a pas une existence distincte et indépendante; certains mots, comme les substantifs, ont une signification propre; tandis que d'autres, comme les propositions, les conjonctions et certains adjectifs, n'ont point une signification propre; leur rôle est de modifier le sens des autres mots; ainsi 
"Le petit Emile , doit-il compter pour un seul mot ou pour trois mots? - Dans la phrase a Si le cheval se brise, le si doit-il compter pour un mot? L'article le doit-il compter pour un mot? Le pronom personnel se doit-il compter pour un mot? On voit combien la question est difficile; nous la posons parce que nous pensons utile d'établir un rapprochement entre la mémoire des mots isolés et la mémoire des phrases. Supposons que nous cherchions à trouver, pour ces deux mémoires différentes, une série équivalente comme longueur; il nous semble que dans ce cas on ne doit tenir compte que des mots ayant une signification propre; ainsi, nous pensons pouvoir comparer comme longueur les deux séries suivantes:

$$
\begin{gathered}
\text { Foin. - Trompette. - Manger. - Cheval. } \\
\text { Le cheval du trompette a mangé du foin. }
\end{gathered}
$$

Les pronoms, les articles, etc., nous paraissent être comparables à des liens, à des sortes de petits clous qui unissent les mots entre eux; et la question réellement intéressante, à ce qu'il nous semble, est celle de savoir isi ces liens logiques qui forment la phrase facilitent le travail de la mémoire, et dans quelle mesure. Dans tout ce qui suit, par conséquent, nous ne tiendrons pas compte du nombre exact de mots contenus dans les phrases; nous diviserons la phrase en un certain nombre de groupes que nous avons marqués et separés au moyen de lignes verticales (voir p. 26). La séparation de ces différents groupes a l'inconvénient, nous le reconnaissons, d'être un peu arbitraire ; un auteur constituera des groupes plus grands, un autre des groupes plus petits. C'est un inconvénient qui nous paraít difficile à éviter.

A propos de la quantité de souvenirs qui restent après l'audition unique d'une phrase, nous examinerons trois questions : les effets produits par la différence d'âge des enfants; les effets produits par la longueur croissante des phrases, la comparaison entre la mémoire des mots isolés et la mémoire des phrases. $11^{\circ}$ Le nombre de mots retenus, après l'audition d'une ou de plusieurs phrases, est en rapport avec l'âge des enfants.

Nous réunissons les résultats les plus importants qui se rapportent à cette question dans le tableau $I$.

Explication du tableau I. - Il contient une expérience de mémoire faite sur les $\mathbf{5}$ classes d'une mène école. Le directeur de l'école a lu lui-même dans toutes les flasses, en cherchant, 
autant que possible, l'uniformité des conditions d'expérience. Les phrases lues ont respectivement 11,14 et 20 mots, et 5,8 , et 8 groupes de mots. Nous avons donné plus haut le texte de toutes les phrases dictées; celles qui ont été lues dans cette école seront faciles à reconnaitre, par l'indication du nombre de mots. L'expérience qui a été faite la première est celle de la phrase de 20 mots ( 8 groupes de mots); ensuite on fait celle de la phrase de 14 mots, et en troisième lieu celle de la phráse de 11 mots.

\section{T A BLEA U I}

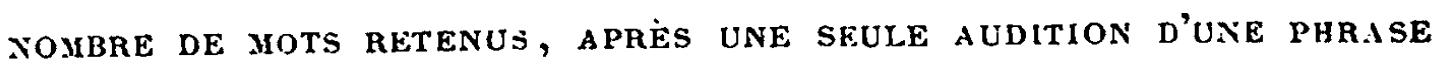

\begin{tabular}{|c|c|c|c|c|c|c|c|c|c|c|}
\hline \multirow{3}{*}{$\begin{array}{c}\text { INDICATION } \\
\text { des } \\
\text { phrases } \\
\text { dictées. }\end{array}$} & \multicolumn{2}{|c|}{$\mathbf{1}^{\mathrm{re}}$ Classe } & \multicolumn{2}{|c|}{2 e CLASSE } & \multicolumn{2}{|c|}{$3^{e} \quad$ CLASSE } & \multicolumn{2}{|c|}{$4^{e}$ CLASSE } & \multicolumn{2}{|c|}{$5^{\mathrm{e}}$ CLASSE } \\
\hline & 35 & élèves & 35 & élèves & 33 & éléves & 33 & lèves & 44 & élèves \\
\hline & 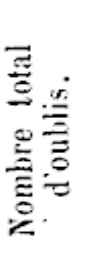 & 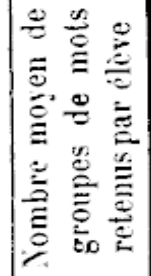 & 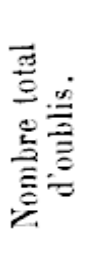 & 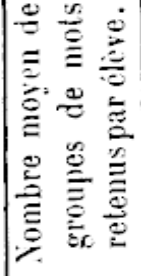 & 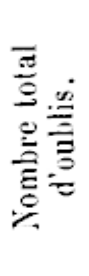 & 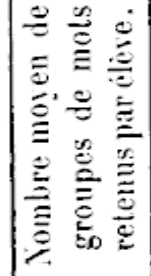 & 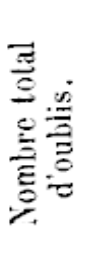 & 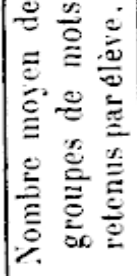 & 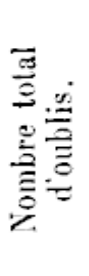 & 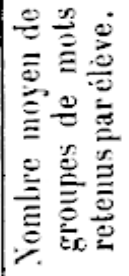 \\
\hline $\begin{array}{l}\text { Phrase de } \\
5 \text { groupes } \\
\text { de mots. } \\
(11 \text { mots }) \text {. }\end{array}$ & 0 & $\ddot{3}$ & 0 & $\ddot{3}$ & 0 & $\breve{~}$ & 3 & 4,9 & 5 & 4,8 \\
\hline $\begin{array}{l}\text { Phrasede } \\
8 \text { groupes } \\
\text { de mots. } \\
\text { (14 mots). }\end{array}$ & 4 & 7,9 & 3 & 7,9 & 3 & 7,9 & 13 & 7,8 & 24 & 7,5 \\
\hline $\begin{array}{l}\text { Phrase de } \\
8 \text { groupes } \\
\text { de mots. } \\
\text { (20 mots). }\end{array}$ & 11 & 7,6 & 29 & $\tau, 1$ & $2 \ddot{3}$ & $\pi, 2$ & $\breve{39}$ & 6,2 & 60 & 6,6 \\
\hline
\end{tabular}

Ce tableau I est surtout destinè à éclairer un point particulier; les différences de mémoire qui tiennent aux différences d'àge ${ }^{1}$. Ces différences, pour une raison ou une autre, ne nous ont pas paru très marquées clans nos expériences sur la mémoire des mots isolés; elles apparaissent un peu plus nettement dans la mémoire des phrases courtes; les différences sont petites, mais constantes.

(1) Age des enfants : $5^{\circ}$ classe, en moyenne 9 ans; $1^{\text {re }}$ classe, en moyenne 12 ans. 
$2^{\circ}$ Le nombre de mots retenus, après l'audition d'une phrase ou d'une série de phrases, augmente avec la longueur des phrases, mais cette augmentation se fait dans des proportions faibles.

Nous avons fait dicter aux élèves des morceaux de longueur différente pour apprécier la quantité des oublis par rapport à la longueur du morceau. Les résultats sont indiqués dans notre tableau II.

\section{TABLEA U I I}

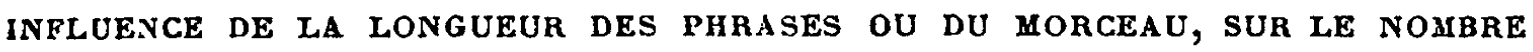

DES OUBLIS, DES SYNONYMES ET DES SUbSTITUTIONS PAR ANALOGIE DE SENS

\begin{tabular}{|c|c|c|c|c|c|c|c|}
\hline & $\begin{array}{c}5 \\
\text { groupes } \\
\text { de } \\
\text { mots } \\
(11 \text { mots })\end{array}$ & $\begin{array}{c}8 \\
\text { groupes } \\
\text { de } \\
\text { mots } \\
(14 \text { mots })\end{array}$ & $\left|\begin{array}{c}8 \\
\text { groupes } \\
\text { do } \\
\text { mots } \\
(20 \text { mots })\end{array}\right|$ & $\begin{array}{c}17 \\
\text { groupes } \\
\text { de } \\
\text { mots } \\
(38 \text { mots })\end{array}$ & $\begin{array}{c}19 \\
\text { groupes } \\
\text { de } \\
\text { mots } \\
(60 \text { mots })\end{array}$ & $\begin{array}{c}24 \\
\text { groupes } \\
\text { de } \\
\text { mots } \\
(74 \text { mots })\end{array}$ & $\begin{array}{c}24 \\
\text { groupes } \\
\text { de } \\
\text { mots } \\
(86 \text { mots })\end{array}$ \\
\hline $\begin{array}{l}\text { Nombre d'é- } \\
\text { lèves. }\end{array}$ & $3 \check{5}$ & 35 & $3 \ddot{3}$ & 33 & 40 & 36 & 36 \\
\hline $\begin{array}{l}\text { Nombre total } \\
\text { d'oublis. }\end{array}$ & 0 & 4 & 11 & 66 & 199 & 213 & 282 \\
\hline $\begin{array}{l}\text { Nombre de } \\
\text { groupes rete- } \\
\text { nus par élève. }\end{array}$ & 5 & 7,9 & $\tau, 6$ & 13 & 14 & 18 & 16,2 \\
\hline $\begin{array}{r}\text { Nombre total } \\
\text { de synonymes. }\end{array}$ & 1 & 9 & 32 & 162 & 149 & 244 & 213 \\
\hline $\begin{array}{l}\text { Nombre total } \\
\text { de substitu- } \\
\text { tions par ana- } \\
\text { logie de sens. }\end{array}$ & 0 & 0 & 4 & 54 & 32 & 42 & 49 \\
\hline
\end{tabular}

Explication du tableau II. - Il exprime les résultats des expériences faites sur la première classe de 4 écoles différentes. Les expériences avec 5 groupes, 8 groupes et pour la seconde fois 8 groupes de mots ont été faites dans une même école; les expériences avec $17,19,24$ et encore 24 groupes, ont été faites chacune dans une école différente; comme toutes ces écoles sont 
soumises à la même discipline, donnent la même instruction et se recrutent dans la mème portion de la population parisienne, nous pensons que les résultats obtenus dans deux écoles différentes sont, dans une certaine mesure, comparables. Ce qui ne l'est pas, ce sont les morceaux dictés ; chaque morceau présente par lui-même une difficulté particulière, et tel morceau, par son style, par son sujet, est plus facile à retenir que tel autre, pour les enfants. Ces considérations nous obligent à quelques réserves dans l'interprétalion du tableau II. On peut cependant remarquer que le nombre des mots retenus augmente avec la longueur des morceaux; mais cette augmentation est relativement assez lente. Ainsi, pour 8 groupes de mots, on oublie une fraction de groupe, soit $\frac{1}{20}$; pour 17 groupes, on oublie 2 groupes soit $\frac{1}{8}$; pour 24 groupes, on oublie 6 à 8 groupes, soit $\frac{1}{3}$ ou $\frac{1}{4}$

$3^{\circ}$ La mémoire verbale des phrases est, dans les cas où nous avons pu faire une comparaison entre les deux, environ vingtcinq fois supérieure à la mémoire des mots isolés.

Il est facile de comprendre comment nous pouvons comparer ces deux mémoires et dire dans quelle mesure l'une est supérieure à l'autre. Supposons un enfant qui, lans une expérience sur la mémoire des phrases, commet une erreur, puis qui, dans une expérience équivalente sur la mémoire des mots isolés, commet dix erreurs; si les deux expériences sont équivalentes, c'est-àdire si le nombre des mots isolés est égal au nombre des groupes de mots, on pourra dire que la mémoire qui donne lieu à une seule erreur est dix fois supérieure à la mémoire qui donne lieu à dix erreurs.

Pour comparer les deux mémoires, il faut comparer le tableau I du présent article, et le tableau I de l'article sur la mémoire des mots isolés; ce rapprochement, il est inutile de le dire, est tout entier à l'avantage de la mémoire des phrases. Un exemple : une phrase de 8 groupes de mots étant lue une fois, les élèves d'une $1^{\text {re }}$ classe n'oublient que $\frac{1}{80}$; si on lit à ces mêmes èlèves une série de 7 mots isolés (il aurait fallu avoir une série de 8 mots, elle nous manque) ils oublient alors $\frac{20}{\mathbf{7 0}}$ de ce qu'on leur lit, soit, pour employer une mesure grossière, ils oublient vingt-cinq fois plus de mots. Donc, pour cette expérience en particulier, la mémoire des phrases est 25 fois supérieure à la mémoire des mots isolés. 
Ces différences nous paraissent être dues à deux causes principales. Lorsqu'on récite devant une personne une série de mots ou une phrase, on provoque dans sa conscience une série d'images. Dans le cas où il s'agit de mots isolés, ce sont des images disparates qui ne sont point associées entre elles naturellement, et que le sujet écoute sans chercher à les associer ; au contraire, dans la phrase, les images sont associées, en conYinuité les unes avec les autres, et parfaitement organisées. Il en résulte qu'au moment de l'acte de'mémoire, on a pour se rappeler la phrase, d'association des idées, et cette association des idées n'opère pas, ou opère beaucoup moins dans le rappel de mots isolés. Ce n'est pas la seule cause de la supériorité que présente la mémoire des phrases.' Lorsqu'on écoute une série de mots isolés, on éprouve une curieuse impression subjective; à mesure qu'on entend un mot, on forme rapidement une image; à peine a-t-on le temps de se rendre compte de cette image qu'on entend prononcer un mot nouveau; on est alors obligé de former une nouvelle image qui expulse la première de la conscience et très probablement a pour effet de l'affaiblir. Donc, dans une série de mots isolés, non seulement les images des mots ne s'associent pas, mais elles entrent en conflit les unes avec les autres et tendent à s'exclure; deux raisons pour que la mémoire des mots isolés soit faible.

\section{III}

D

Dans la memolre d'une plirase ou d'une série de prirases, Les MOTS QUI JOUENT LE RÔLE LE PLUS IMPORTANT DANS LE RÉcIT SONT LES MIEUX CONSERVÉS.

Nous ne nous sommes occupés, jusqu'ici, que du nombre total de mots retenus par les élèves, aprèsl'audition d'un ensemble de phrases. Nous allons maintenant examiner comment les oublis sont répartis dans cet ensemble.

Nous avons figuré cette répartition dans le tableau graphique I, donné ici comme échantillon.

Explication du tableau graphique I. - A la base de ce tableau, sur la ligne des abscisses, nous avons écrit le morceau de prose à retenir, en divisant ce morceau par petits fragments qui nous paraissent avoir une certaine unité de sens; nous avons discuté plus haut la manière dont il faut établir ces fragments. 


\section{TABLEAU GRAPHIQUE I}

DISTRIBLTION DES ERREURS DANS LES DIFFÉRENTES PARTIES D'UN RÉcit REPRODUIT DE MÉmoIRE, APRÈS UNE SEULE AUdition, PAR UNE CLASSE DE QUARANTE ÉLĖVES.

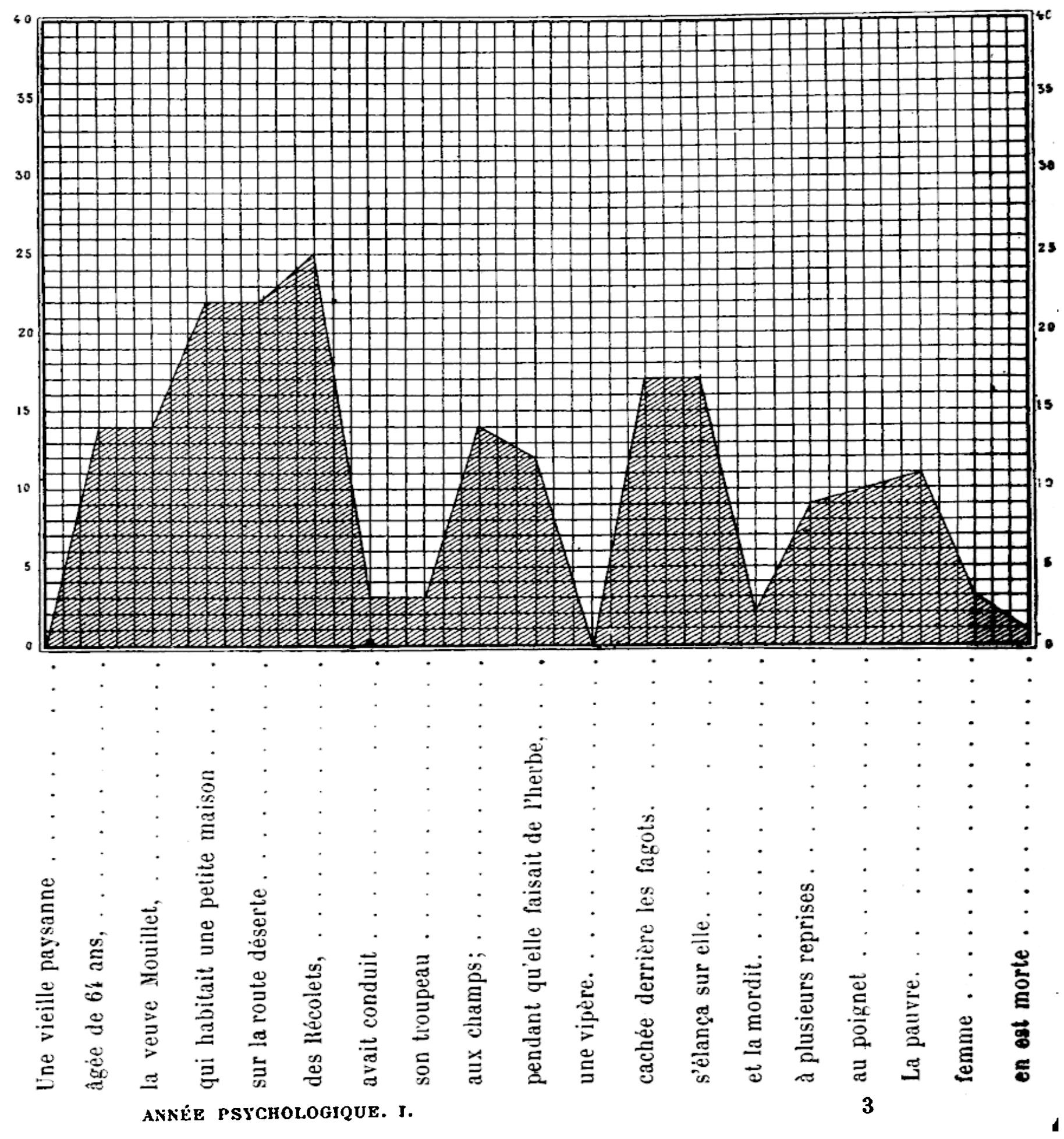


Sur la ligne des ordonnées, en regard de chaque membre de phrase, nous avons marqué par quel nombre d'élèves ce membre de phrase a été oublié; le nombre d'élèves composant la classe dans laquelle on fait l'expérience se trouve indiqué sur la première ordonnée; il a été dans le graphique de 40 ; par exemple, 14 élèves ont oubliẻ le membre de phrase «âgée de 64 ans \$; dans ce cas, on marque sur la seconde ordonnée, correspondant à ce membre de phrase, un point à la hauteur de 14. On répète cette opération pour les différents membres de phrase composant le morceau appris, on réunit ces points par des lignes droites, et on noircit toute la surface comprise entre ce tracé et la ligne des abscisses. On obtient ainsi une surface rectangulaire, divisée, par une ligne sinueuse, en deux parties inégales, l'une noire et l'autre blanche; le rapport entre ces deux parties correspond au rapport entre les oublis et les souvenirs conservés. Il faut ajouter que nous entendons ici par oublis tous les mots et toutes les parties de phrases qui ne sont pas conservés intacts, et aussi tous ceux qui ne sont pas remplacés par d'autres mots d'un sens analogue ou mème d'un sens différent; l'oubli est donc pris ici dans le sens d'une lacune de mémoire que rien ne vient rem. plir.

Le premier coup d'œil jeté sur le graphique du tableau I montre que les oublis ne se répartissent pas d'une manière uniforme sur toute la ligne de la phrase ou du morceau de prose : le profil de la surface noire du graphique est formé de lignes brisées qui dessinent tantôt des caps, tantôt des golfes, indiquant ainsi que dans des membres de phrases qui se suivent, le nombre de fois que les mots ont été oubliés varie continuellement avec la nature de ces mots. A certains endroits un cap noir s'avance presque jusqu'au haut de la région blanche du graphique, ce qui exprime que le membre de phrase correspondant a toujours ou presque toujours été oublié, tandis qu'à d'autres endroits la surface noire présente une entaille blanche qui atteint la ligne inférieure de l'abscisse ; la phrase placée en ce dernier endroit n'a jamais été oubliée.

On pourrait croire que comme ces contours présentent une grande irrégularité et une grande différence d'un graphique à l'autre, on ne peut y chercher aucune constance de forme, et que par conséquent, il n'y a pas de règle à établir relativement à la distribution des oublis. Ce serait une erreur. Cette distribution des oublis varie avec les phrases; mais pour une même phrase elle présente une grande uniformité. En voici deux 
preuves principales. Nous avons fait dicter le même morceau de prose dans des écoles différentes, et en faisant ensuite le graphique des résultats, nous avons des tableaux tout à fait analogues dans lesquels la quantité des oublis varie, mais où la 'répartition relative de ces oublis reste sensiblement la méme. La place nous manque malheureusement pour donner ces résultats en graphique; nous les donnons plus loin en chiffres (tableaux III i IX). - En second lieu, si on laisse écouler 20 jours après l'expérience et que retournant dans la même classe on fasse ¿crire aux élèves ce qu'ils se rappellent de la phrase dictée ${ }^{1}$, le "raphique de cette seconde expérience ne diffère guère de celui le la première que par la quantité totale des oublis; la répartition, ici encore, reste la même, comme le montre la table de chiffres ci-jointe (tableaux III à V).

Il y a donc dans ces résultats, pour qui sait les lire, une fixité remarquable dont nous devons maintenant rechercher la signification. La répartition des oublis est due, comme nous allons le voir, au sens de la phrase. Le sens de la phrase est bien dislinct du sens des mots ; il ne résulte pas d'une simple addition du sens des mots. Il y a dans une phrase des parties importantes, et si essentielles à la phrase que si on les supprimait, la phrase n'aurait plus de sens : il y a également des parties accessoires, qui modifient le sens principal, mais qui ont une importance moindre, car on pourrait les supprimer sans rendre la phrase inintelligible. Or, il est bien facile de se rendre compte que c'est la partie principale, essentielle de la phrase qui est la mieux retenue.

Ainsi, prenons le récit mis en graphique : Une vieille paysanne... etc., récit que nous avons donné intégralement plus lıaut. Nous avons pris ce récit dans les faits divers d'un journal, et nous l'avons légèrement modifié, en le chargeant de phrases incidentes qui ne présentent pas grande importance. Qu'on exaunine, dans le graphique I, les membres de phrase où la surface noire est le plus entamée par la surface blanche; ce sont les points où les oublis ont été les moins nombreux; on aura ainsi lesphrases suivantes : une vieille paysanne - avait conduit son troupeau - une vipère - la mordit - la femme - est morte. Or, c'est là précisément, la partie essentielle du récit ; le reste n'est qu'ac-

(1) Au moment de la première dictée, nous n'avions pas l'idée de cette répétition après vingt jours d'intervalle, et par conséquent les élèves n'en étaient pas avertis d'avance. 
cessoire, et beaucoup moins important. En faisant la même opération sur le tableau VIII relatif au récit d'un commencemen! d'incendie, récit que nous avons donné plus haut in extenso, on a un résultat analogue : Un commencement d'incendie - s'esı déclar'é - dans l'appartement - de I $^{\text {me }}$ Becquet - qui tenait ure bougie. - Les pompiers. Ges fragments de phrases nf forment pas un récit complet ; ils indiquent seulement les points où la mémoire a été le plus profondément impressionnée, et en réalité, ce sont les points les plus importants de l'histoire. Fgale. ment pour le récit du tableau VI, les parties les moins oubliées sont les suivantes : Imprudence d'enfants. - Plusieurs enfants s'amusaient - machine ì mortier - l'un d'eux, Victor Anti. quet - eut sa main - écrasée. - Il a recu - dans une pharmacie - chez ses parents. Le nombre des fragments de phrases bien retenus est ici plus considérable, parce que le morceau est moins long; mais on remarque que ce sont les tragments les plus importants. Enfin, pour terminer, nous citerons une phrase moins longue que les précédentes, composée seulement de 20 mots : Le petit Emile, etc. Cette phrase a été lue dans les quatre classes d'une école; voici à quoi elle se résume pour la première classe : Le petit Émile - a obtenu - un chevalmécanique - en récompense - de sa bonne conduite - à l'école.

La dernière classe donne : Le petit límile - a obtenu - un cheval mécanique. Ici, la réduction est plus considérable.

( On voit donc que la mémoire des mots et des idées, que nous pouvons étudier dans ces expériences, réduit le récit entendu en opérant un choix les parties essentielles du récit sont beaucoup mieux retenues que les autres.

$\mathrm{Or}$, il faut remarquer que l'état de la mémoire nous renseignt ici d'une manière indirecte sur l'étal de la perception, que la mémoire reproduit; les mots qui sont les mieux retenus, et qu: sont précisément ceux qui présentent le plus d'importance. sont incontestablement ceux qui ont éveillé, au moment de l'au. dition, le maximum d'attention : d'où nous pouvons conclure que lorsqu'on entend une phrase prononcée, qu'on l'écoute el qu'on la comprend, il est très probable que l'attention ne reste pas dans un état uniforme d'activité ${ }^{1}$; elle est plus fortemen1 éveillée à certains moments, moins fortement à d'autres. Il st produit du reste un phénomène psychologique tout à fait ana.

(1) Ceci est démontré par un grand nombre de faits. Voir James, Psy chology, 1, \&20. 
TABLEAU III. - INFLUENCE DU SENS DES MOTS SUR LES OCBLIS

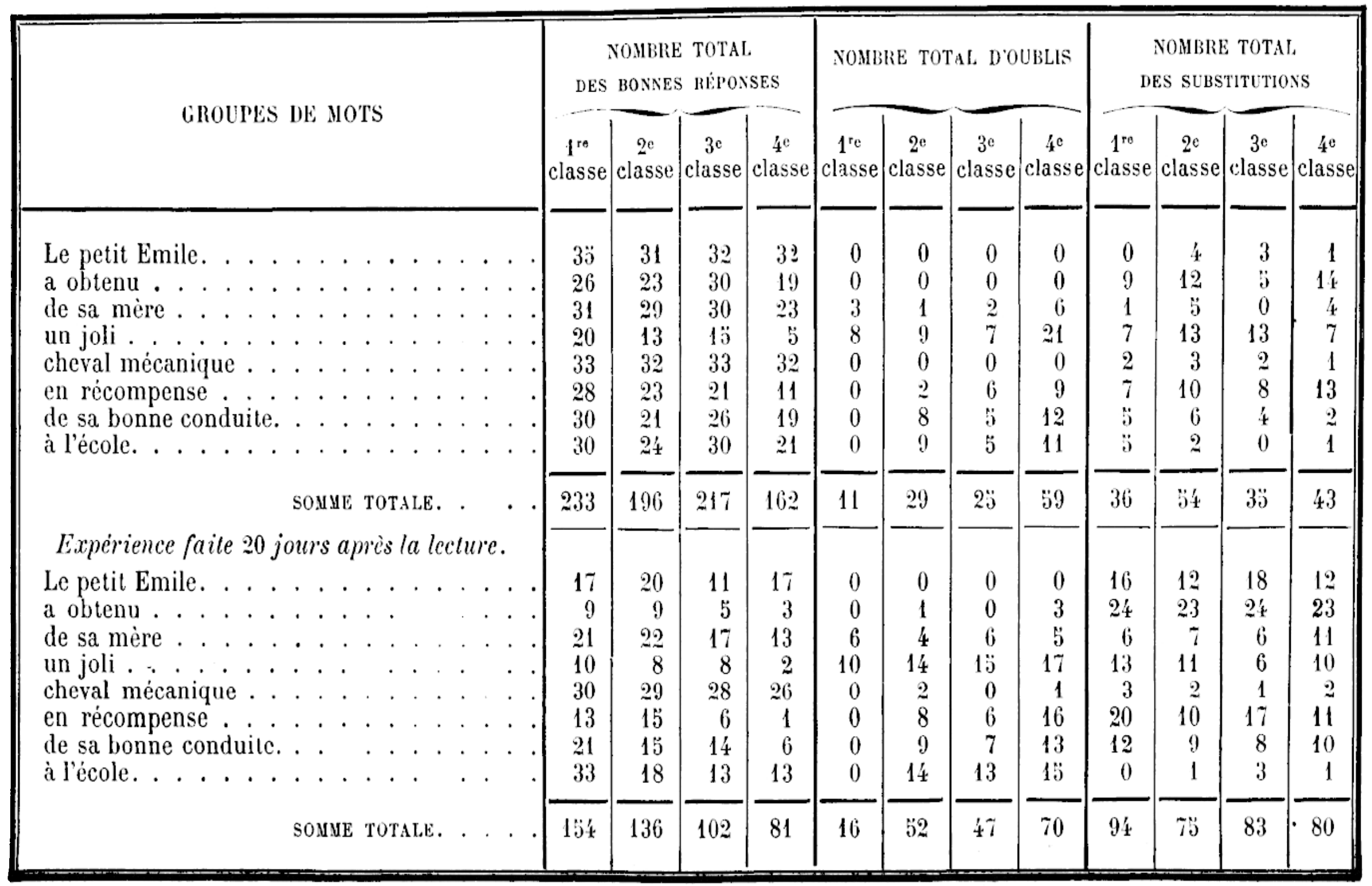


TABLEAU IV. - INFLUENCE DU SENS DES MOTS SUR LES OUBLIS

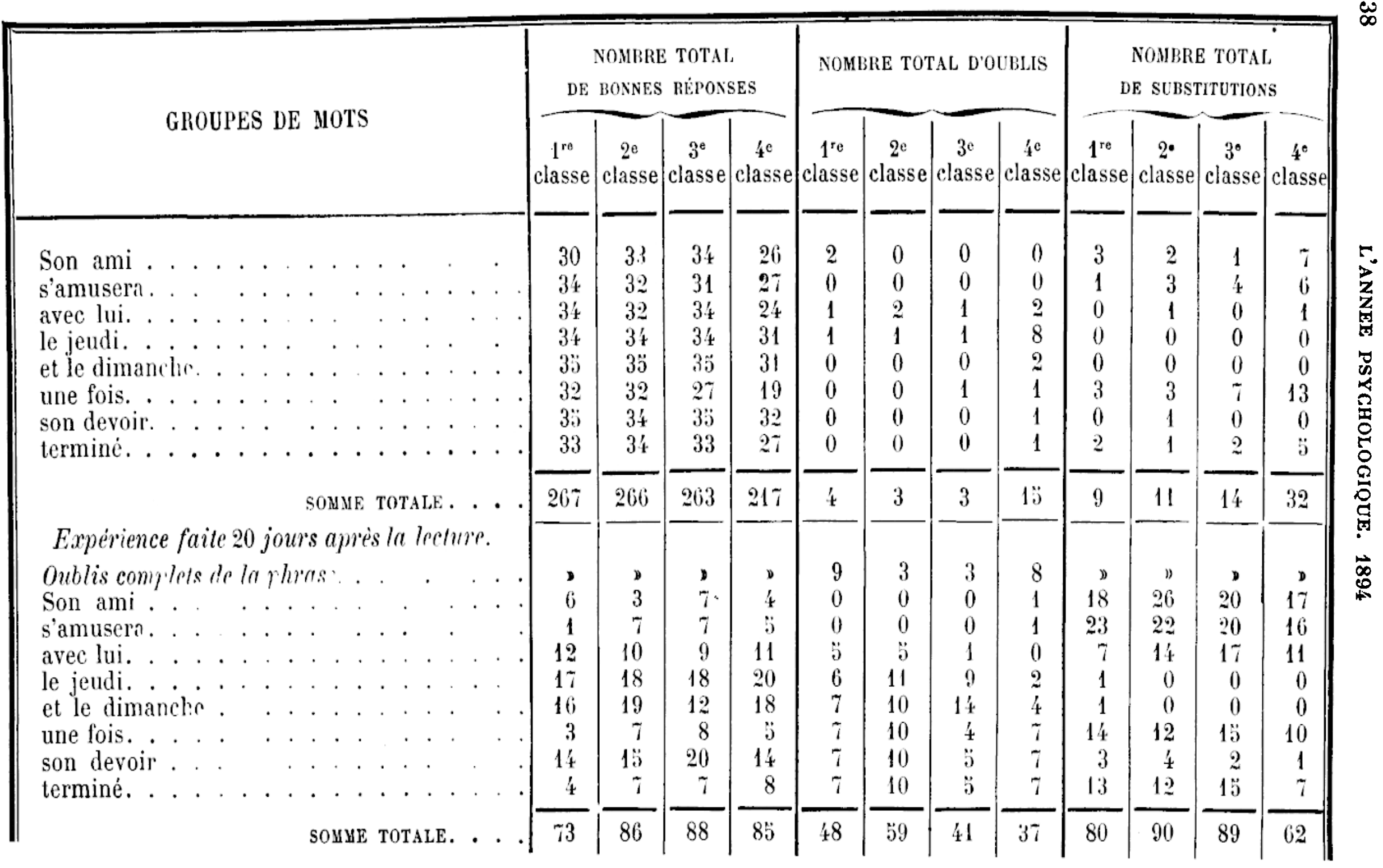


TABLEAU V. - INFLUENCE DU SENS DES MOTS SUR LES OUBLIS

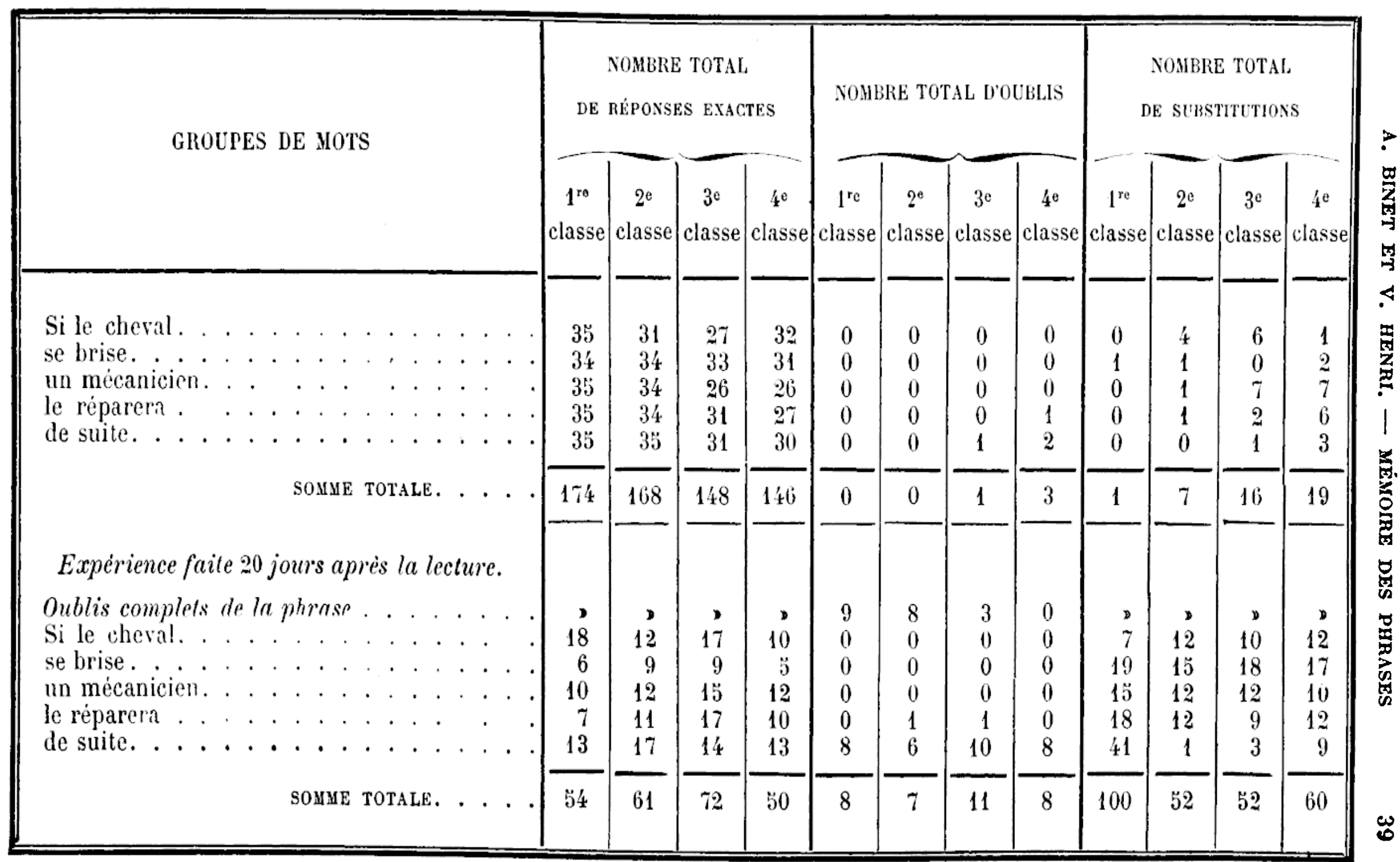


TABLEAU VI. - INFLUENCE DU SENS DES MOTS SUR LES OUBLIS

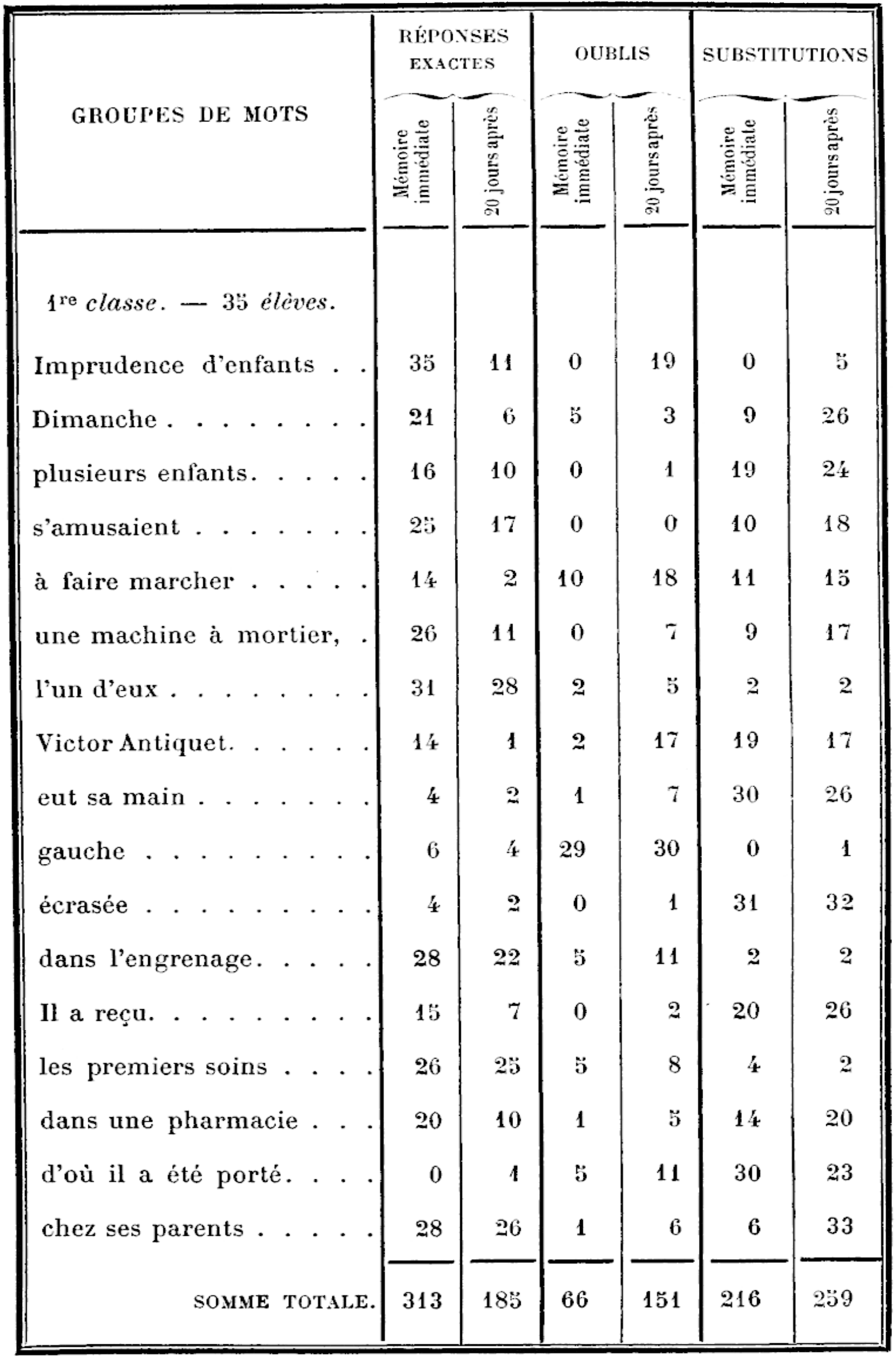


A. BINET ET V. HENRI. - MÉMOIRE DES PHRASES

TABLEAU VII. - INFLUENCE DU SENS DES MOTS SUR LES OCBLIS

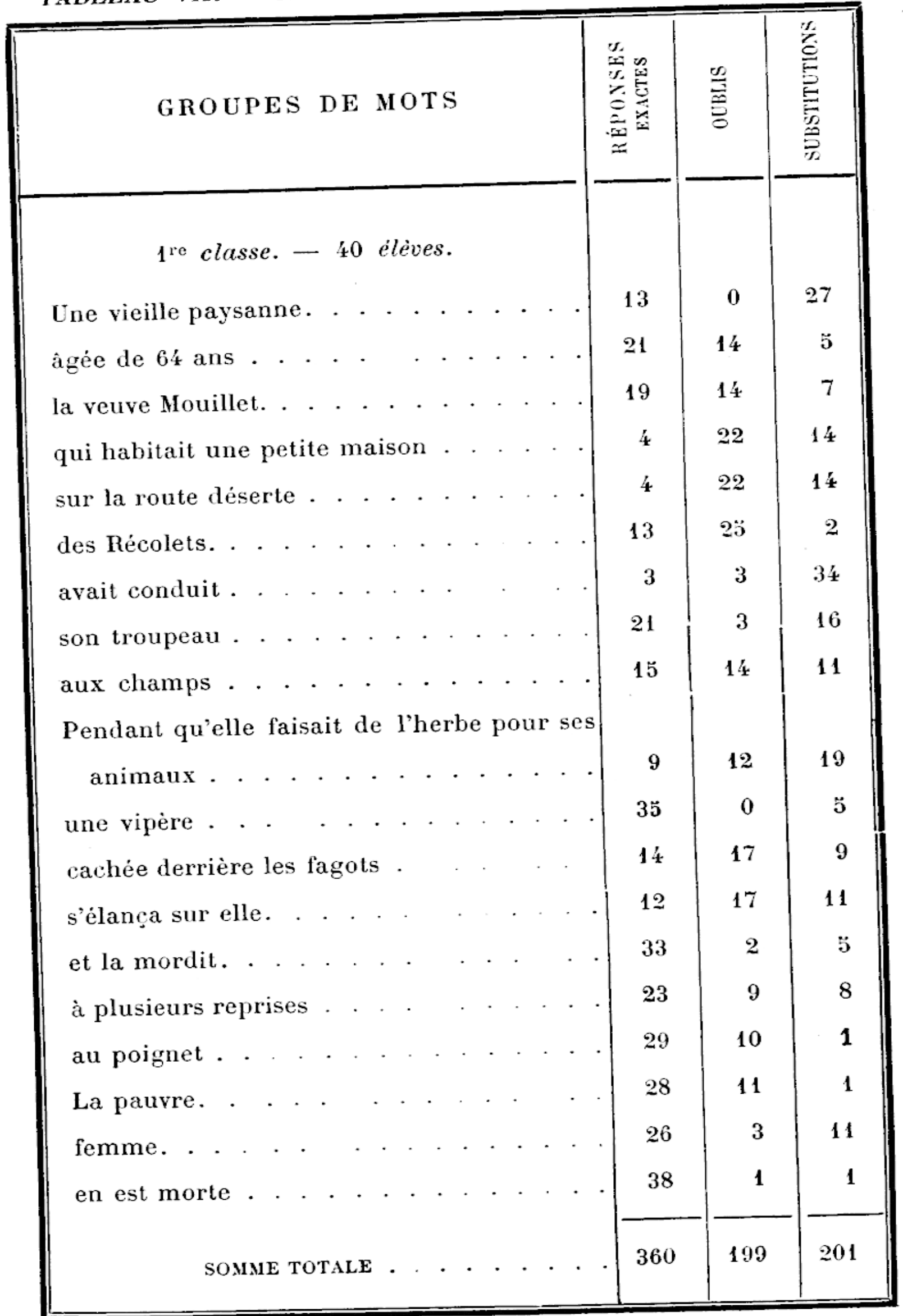


TABLEAU VIII. - INFLUENGE DU SENS DES MOTS SUR LES OUBLIS

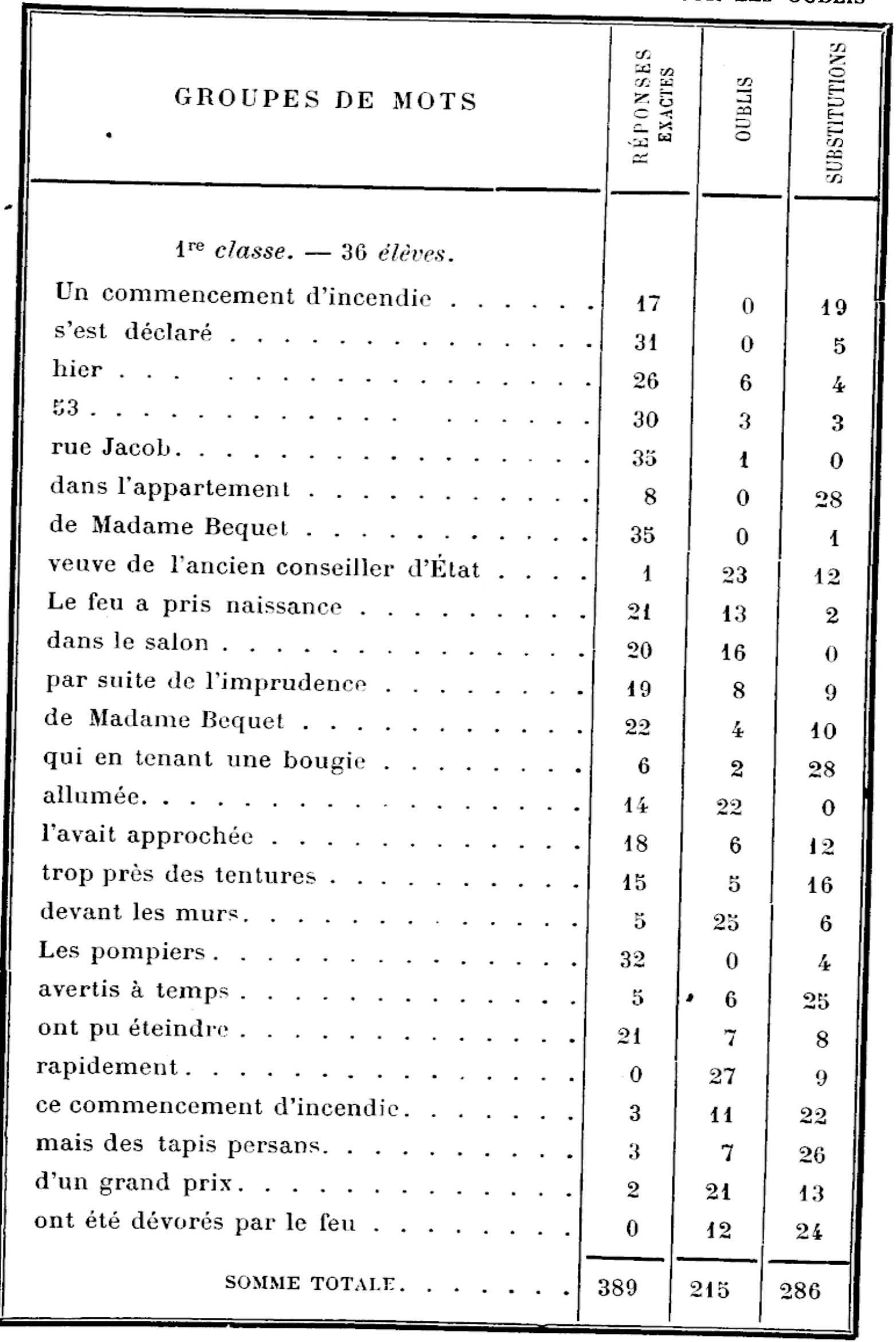


A. BINET ET V. HENRI. - MENOIRE DES PHRASES

TABLEAU IX - INFLUENCE DU SENS DES MOTS SUR LES OUBLIS

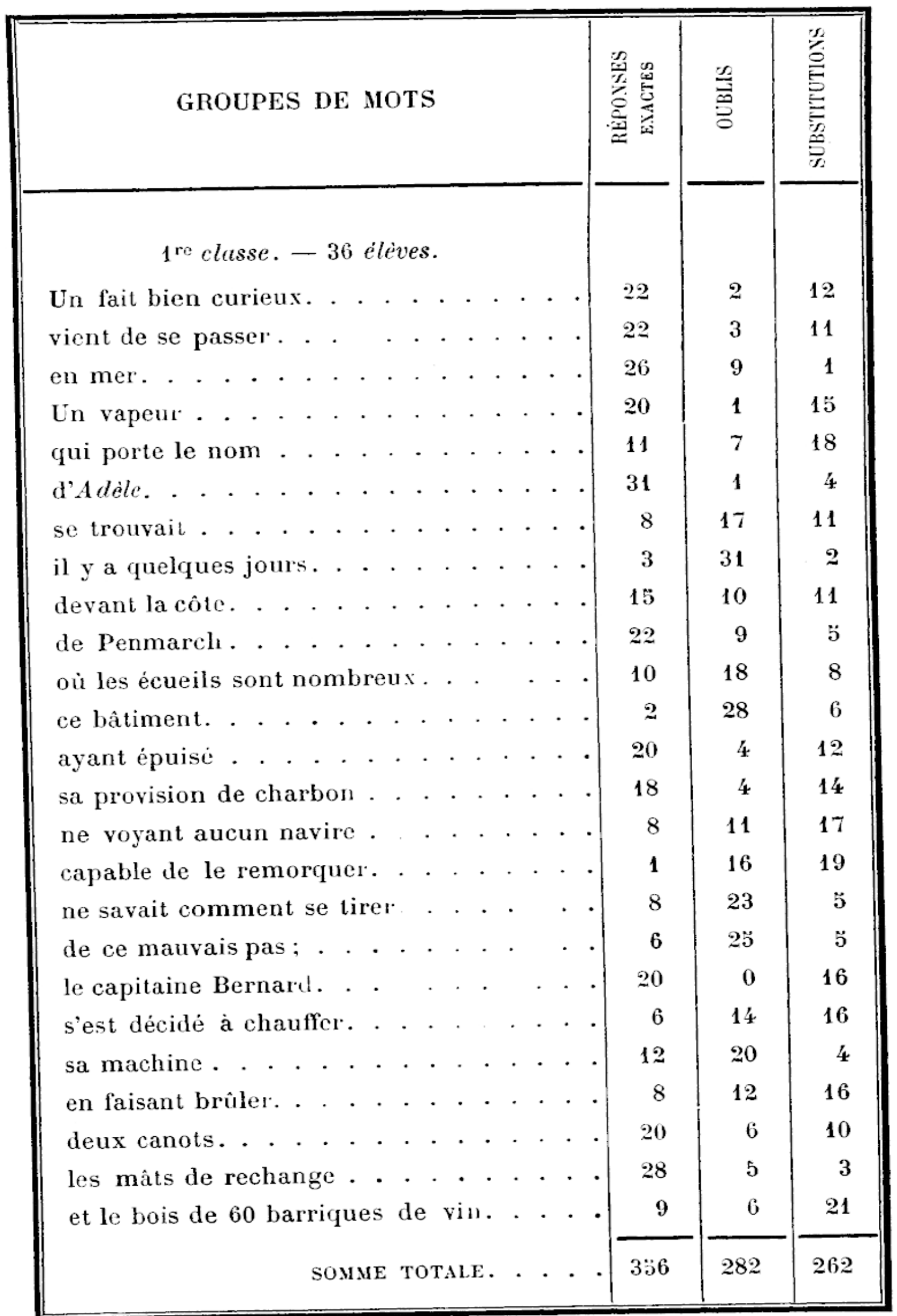


logue quand on prononce une phrase d'un ton naturel, pour exprimer une pensée; on allonge la durée des mots importants, et on prononce plus rapidement les mots accessoires et insignifiant, ainsi que le montre l'enregistrement graphique de la parole au moyen d'un appareil quelconque ${ }^{1}$.

Ce n'est pas tout; nos tableaux ne nous donnent pas seulement un instrument d'analyse permettant de distinguer l'essentiel de l'accessoire; nous pouvons voir, pour les parties qui ne sont qu'accessoires, dans quelle mesure, et avec quel degré elles le sont; il est bien évident, par exemple, qu'un mot sur lequel se font 10 oublis a une importance relativement plus grande qu'un mot sur lequel se font 20 oublis; le degré est différent. Or, nous voyons sur nos graphiques que chaque membre de phrase a sa hauteur particulière, son nombre d'oubli correspondant; c'est là ce qui donne à l'ensemble des courbes un aspect si irrégulier. Si on réfléchit que ce nombre d'oublis qui se produit dans chaque cas marque l'importance relative de chaque mot et le degré d'attention qu'il éveille, il en résulte que nous pourrons mesurer par ce moyen l'intensité psychologique de la succession d'états de conscience qui correspondent à une phrase donnée. C'est là, qu'on le remarque bien, l'incontestable avantage d'une étude collective; si on fait une expérience individuelle de mémoire, cette experience ne peut montrer qu'une chose : des parties sont oubliées, d'autres sont retenues. Dans l'expérience collective, au contraire, on voit avec quel degré les parties oubliées le sont ; c'est une dynamométrie de l'attention.

Or, les tracés nous amènent à cette constatation que le nombre des oublis varie d'un mot ou d'un groupe de mots à l'autre, en présentant des écarts souvent très appréciables, et nous pouvons en conclure que pendant l'audition d'une phrase l'état de l'attention subit continuellement des changements de - force; en d'autres termes, les images mentales éveillées présentent des modifications incessantes d'éclat.

Pour donner une expression graphique à ces résultats, nous imprimons ici en caractères différents le texte des récits dictés aux élèves; l'importance typographique des caractères est chargée de représenter l'importance psychologique des mots; c'est une manière un peu différente de matérialiser les résultats déjà figurés dans nos tableaux graphiques.

(1) Voir Binet et Ilenri, Les actions d'arrêt dans les phénomènes de la parole, Rev. Phil. 1894, p. 608-620. 
$1^{\circ}$ Résultat de la $1^{\mathrm{re}}$ classe :

Le petit Émile a obtenu de sa mère un joli cheval mécanique en récompense de sa bonne conduite à l'école.

$2^{\circ}$ Résultat de la $4^{\circ}$ classe:

Le petit Émile a obtenu de sa mère un joli cheval mécanique en récompense de sa bonne conduite à l'école.

$3^{\circ}$ Résultat de la $1^{\mathrm{re}}$ classe :

Son ami s'amusera avec lui le jeudi et le dimanche une fois son devoir terminé.

$4^{\circ}$ Résultat de la $4^{\mathrm{c}}$ classe :

Son ami s'amusera avec lui le jeudi et le dimanche une fois son devoir terminé.

$5^{\circ}$ Résultat de la $1^{\text {re }}$ classe :

Si le cheval se brise un mécanicien le réparera de suite.

$6^{\circ}$ Résultat de la $4^{e}$ classe :

Si le cheval se brise un mécanicien le réparera de suite.

$7^{\circ}$ Résultat de la mémoire immédiate :

Imprudence d'enfants. Dimanche plusieurs enfants s'amusaient à faire marcher une machine à mortier, l'un d'eux, Victor Antiquet, eut sa main gauche écrasée dans l'engrenage. Il a reçu les premiers soins dans une pharmacie d'où il a été porté chez ses parents. 
$8^{\circ}$ Résultat de la mémoire du morceau après 20 jours :

Imprudence d'enfants. Dimanche plusieurs enfants s'amusaient à faire marcher une machine à mortier, l'un d'eux, Victor Antiquet eut sa main gauche écrasée dans l'engrenage. Il a reçu les premiers soins dans une pharmacie d'où il a été porté chez ses parents.

$9^{\circ}$ Une vieille paysanne âgée de 64 ans qui habitait une petite maison des Récolets avait conduit son troupeau aux champs. Pendant qu'elle faisait de l'herbe pour ses animaux une vipère cachée derrière les fagots s'élança sur elle et la mordit à plusieurs reprises au poignet. La pauvre femme en est morte.

10' Un commencement d'incendie s'est déclaré hier 53, rue Jacob, dans l'appartement de $M^{\text {me }}$ Béquet, veuve de l'ancien conseiller d'État. Le feu a prisınaissance dans le salon par suite de l'imprudence de $M_{i}^{m}$ Béquet qui en tenant une bougie allumée l'avait:approchée trop près des tentures sur les murs. Les pompiers avertis à temps ont pu éteindre rapidement ce commencement d'incendie, mais des tapis persans d'un grand prix ont été dévorés par le feu.

11. Un fait bien curieux vient de se passer en mer, un vapeur qui porte le nom d'Adèle se trouvait il y a quelques jours devant la côte de Penmarch où les écueils sont nombreux; ce bâtiment ayant épuisé sa provision de charbon ne voyant aucun autre navire capable de le remorquer, ne savait comment se tirer de ce mauvais pas; le capitaine Bernard s'est décidé à chauffer 
sa machine en faisant brûler deux canots, les mâts de rechange et le bois de soixante barriques de vin.

M. William James, dans ses Principes de Psychologie', a quelques pages où il traite la question du cours de la pensée (The stream of thought), et il a montré au moyen de schémas ingénieux les changements d'intensité qui se produisent dans les états de conscience exprimés par une phrase qu'on prononce ou qu'on entend prononcer.

Nous sommes heureux de constater que ces vues théoriques se trouvent confirmées par nos expériences ; celles-ci montrent en outre que les changements d'intensité sont moins simples que M. W. James ne les a supposés. Dans les schémas qu'il a construits, et qui rappellent un peu les tracés de nos tableaux, les lignes sont arrondies, et montent graduellement jusqu'à une hauteur maxima, sans ces écarts brusques et ces irrégularités que nous constatons dans nos expériences de mémoire. Rien n'est plus curieux que de comparer la courbe dessinée par hypothèse avec la courbe donnée par la réalité.

Il nous reste maintenant à faire l'examen attentif de chacun des mots composant le récit dicté aux élèves, pour rechercher quelles sont les qualités de chaque mot qui ont réussi à assurer sa survivance dans la mémoire.

Nous avons supposẻ implicitement jusqu'ici que c'est le sens du mot qui exerce l'influence principale; ou plutôt, poür parler plus exactement, ce n'est pas le sens spécial de chaque mot qui importe, mais pien le rôle qu'il joue dans la phrase ; il est évident qu'un même mot, le mot maison par exemple, peut dans certaines phrases être très important, et se írouver placé en pleine lumière, tandis que dans d'autres il restera dans l'ombre d'une proposition incidente qui manquera tout à fait d'importance. C'est donc le rôle des mots dans la phrase qui est ici à considérer. Examinons à ce point de vue nos principaux récits.

Les mots le plus souvent oubliés, et qui correspondent aux caps noirs les plus élevés de nos tracés, sont les suivants : Dans la phrase : "Le petit Émile... etc. "l'oubli maximum porte sur le qualificatif joli; on a dicté " un joli cheval mécanique ; des deux épithètes, l'une, celle de mécanique, a toujoursété retenue;

(l) T. 1, p. 223-290. 
l'autre, joli, a été presque toujours oubliée; la différence est extrêmement nette, et peut, semble-t-il, s'expliquer facilement; l'une des épithètes est très significative, tandis que l'autre n'a pour ainsi dire aucun sens, et n'ajoute rien à l'idée. Dans le récit : Un commencement d'incendie... etc. », nous trouvons également que les oublis les plus nombreux ont porté sur des mots qui n'ajoutent aucun sens important à la phrase; dans cette proposition : "qui, en tenant une bougie allumée " l'acte de tenir une bougie, acte essentiel dans le récit, a été bien retenu; l'adjectif allumée, qui est un pléonasme, puisqu'il est dit que le feu a été mis avec la bougie, cet adjectif est presque toujours oublié. Un peu plus loin, il est dit c les pompiers... ont pu éteindre rapidement le feu s; l'adverbe rapidement a été oublié par un grand nombre d'élèves. Ces mots el quelques autres qu'on trouvera en lisant les tables présentaient ce désavantage de ne pas offrir un sens précis; ce sont des mots parasites.

11 est d'autres mots dont l'oubli paraît être dû à des causes un peu différentes; ce sont des mots ayant un sens précis, apportant â l'esprit un détail particulier; mais ce détail ne fait pas partie nécessaire de la phrase: ils n'est pas nécessaire, en quelque sorte, à sa togique.: Parr exemple dans le récit * Imprudence d'enfants... , il est dit que l'un des enfants eut sa main gauche écrasée; l'adjectif gauche a évidemment par lui-même un sens bien net, mais on peut dire que le détail est peu important; que l'enfant ait eu sa main gauche écrasée ou sa main droite, la chose n'est guère intéressante. Aussi l'adjectif a-t-il présenté un nombre d'oublis considérable; de tout le récit, c'est le mot qu'on a le plus oublié. - De même, dans quelques-uns de nos récits nous avons introduit des propositions incidentes, qui par elle-mêmes ont un sens, mais dont le sens ne joue pas un role important dans la phrase; par exemple, dans le récit : « Une vieille paysanne, etc. nous avons placé cette incidente superflue : c qui habitait une petite maison sur la route déserte des Recolets ; le récit n'a rien à faire avec l'adresse de la bonne femme; il importe peu qu'elle habite ici ou là; le fait important, c'est qu'elle a été mordue par une vipère. L'attention des enfants a glissé sur la phrase incidente, qui a donné lieu à un très grand nombre d'oublis. De même encore, dans le récit : « Un commencement d'incendie , l'incidente : "veuve d'un ancien conseiller d'Etat $\bowtie$, qui n'a point de rapport avec le fait même de l'incendie et qui du reste a sans doute été peu comprise, a été 
A. BINET ET V. HENRI. - MÉMOIRE DES PHRASES

très souvent oubliée. Nous arrêtons là nos commentaires; la simple lecture de nos tables suffit amplement à donner d'autres détails.

\section{V}

\section{LES SYNONYMES}

$1^{\circ}$ Le nombre des synonymes employés dans lacte de mémoire est plus grand que le nombre des oublis complets pour les morceaux courts, et plus petit pour les morceaux longs.

- Đańs ce qui précède, nous n'avons tenu compte que des oublis complets, consistant en ce que des mots non seulement ne sont pas reproduits, mais encore ne sont pas remplacés par d'autres mots; souvent, le mot oublié est remplacé par un autre, qui ne figurait pas dans la dictée et qui est par conséquent de l'invention de l'enfant. Nous nous proposons maintenant d'examiner quelle est la nature de ces substitutions.

Ces substitutions sont de plusieurs genres; nous nous oceuperons d'abord de celle qui nous paraît la plus importante par le nombre : ce sont les synonymes. Nous entendons par là, on le comprend, des mots remplaçant ceux de la dictée, qui ont le même sens que ces derniers, mais qui appartiennent à un autre vocabulaire : ainsi s'amuser est remplacé par jouer; c'est le même sens, ce n'est pas tout à fait le même style; ou bien à plusieurs reprises est remplacé par plusieurs fois. Voilà ce que nous appelons des synonymes. Dans nos expériences de mémoire sur les enfants, ces substitutions par synonymes sont très nombreuses. Pour apprécier leur importance, il faut se reporter au tableau II, où le nombre des synonymes employés par les élèves, pour chaque morceau dicté, a été compté dans les différents exercices de mémoire auxquels on les a soumis. Le nombre des synonymes varie, non seulement avec la longueur des morceaux, mais arec le style et la nature du sujet; c'est une observation que nous avons déjà présentée à propos du nombre des oublis; il faut la répéter ici. Les différents exercices de mémoire avec des morceaux de $\mathbf{s}$ à 2'. groupes de mots, que nous indiquons au tableau II, ne sont par conséquent pas comparables. On peut cependant remarquer, sans cuurir le risque de se tromper beaucoup, que le nombre des synonymes employés augmente avec la longueur du morAXXEE PSYGHOLOGIQLE. I. 
ceau; il est de 1 seulement pour un morceau de 5 groupes de mots; il s'élève à 149 pour un morceau de 19 groupes, il atteint 244 pour un morceau de 24 groupes. Cette augmentation du nombre des synonymes dans la répétition de mémoire est la chose du monde la plus naturelle; elle est la preuve que la mémoire verbale, c'est-à-dire que la mémoire des mots exactement entendus, diminue à mesure que le morceau à retenir s'allonge, et que d'autre part la mémoire des idées y supplée ; on ne se rappelle plus les mots, on se rappelle l'idée, et pour l'exprimer on invente des mots, par conséquent on emploie des synonymes.

L'examen du tableau II nous montre un second fait curieux; c'est que pour les morceaux courts le nombre des synonymes est beaucoup plus considérable que le nombre des oublis complets; ainsi, pour les premiers morceaux, il y a 29 synonymes pour 11 oublis complets; quand le morceau dicté est beaucoup plus long, le nombre des synonymes croît, mais il croît moins vite que le nombre des oublis; et pour un morceau assez long, composé de 24 groupes de mots, on voit que le nombre des synonymes est seulement de 213 tandis que le nombre d'oublis est supérieur, de 282 ; il est bien probable que si on allongeait encore le morceau dicté, cette supériorité des oublis complets s'accuserait davantage. Nous croyons ces résultats très simples et très faciles à comprendre. Les synonymes marquent la disparition de la mémoire verbale, et la conservation de la mémoire des idées; les oublis complets marquent la disparition de la mémoire des idées; or celle-ci doit présenter d'autant plus de lacunes que le morceau à retenir est plus long.

On peut, en somme, exprimer la succession de ces phénomènes dans un schéma très simple qui montre que la courbe des oublis complets reste longternps au-dessous de la courbe des synonymes, et qu'elle la dépasse ensuite.

$2^{\circ}$ L'enfant, en reproduisant de mémoire une phrase ou une série de phrases, a une tendance d̀ remplacer les mots entendus par des synonymes appartenant ì son langage familier.

Nous en avons assez dit sur le nombre des synonymes; parlons maintenant de leur qualité. Quand les enfants remplacent un mot ou un groupe de mots par des synonymes, il y a parmi ces synonymes un mot qui obtient une majorité considérable. Ainsi dans une dictée figurent les mots : « la vipère s'élanca sur elle $\diamond ;$ pour des raisons que nous indiquerons plus loin, ce verbe s'élança a été remplacé par plusieurs syno- 


\section{TABLEAU GRAPHIQUE II}

NOMBRE DES OUBLIS, DES SYNONYMES ET DES SUBSTITUTIONS PAR ANALOGIE DE SENS QUi SE PRODUISENT DANS LA RÉpétition, Par LA MÉmoIRE, DE RÉGITS DE DIFFÉRENTES LONGUEURS.

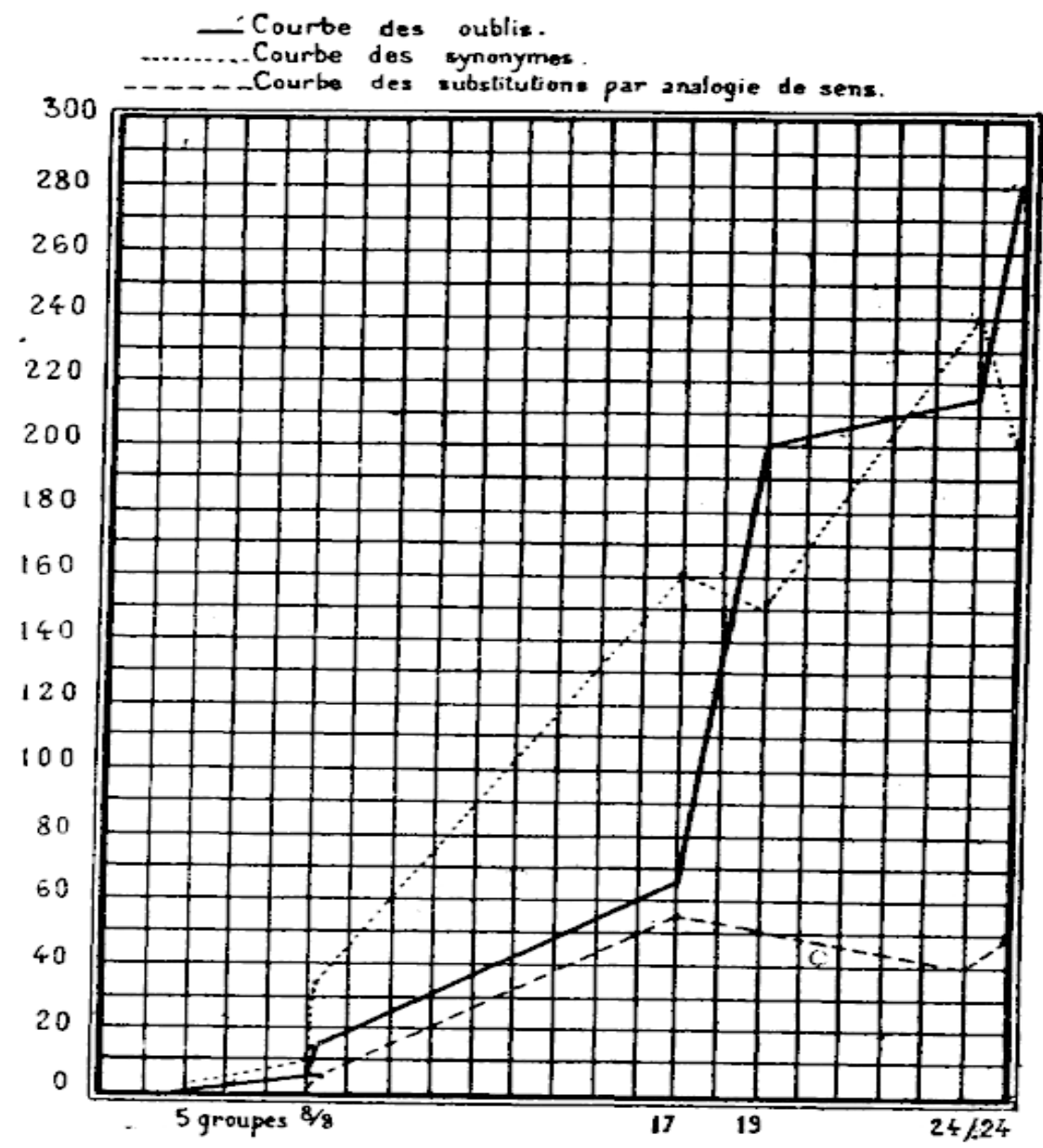

N. B. - Sur la ligne des abscisses sont indiqués les nombres de groupes de mots à retenir (à noter que deux expériences ont été faites avec 8 groupes, deux aussi avec 24 groupes); sur la ligne des ordonnées sont indiqués les nombres d'erreurs. 
nymes différents; nous en comptons trois, se dressa, se jeta et sauta. Or, de ces trois synonymes, sauta est employé 8 fois; se jeta n'est employé que 1 fois, et se dressa, 2 fois. La majorité est donc bien évidente pour le premier de ces verbes.

Il arrive parfois qu'un seul et même synonyme est employé par tous les èlèves qui ont oublié le mot exact. Ainsi, dans le récit amprudence d'enfants , il est dit : * plusieurs enfants s'amusaient >; le verbe s'amuser a été remplacé 10 fois par jouer, et c'est là le seul synonyme employé.

Si on regarde de près les mots pris comme synonymes par les enfants, on remarque qu'ils ont tous un caractère bien particulier; ce sont des mots appartenant au langage familier, ou, pour micux dire, au langage enfantin. Les enfants ont une tendance à remplacer le mot du texte dicté, quand ce mot appartient à un style un peu relevé, par un autre mot dont ils ont darantage l'habitude, et qui se rencontre plus souvent dans leur bouche. Leur acte de mémoire s'accompagne d'un acte de traduction. Ils impriment à la phrase qu'ils font pénétrer dans leur esprit le cachet de leur personnalité, ils la font leur, ils lui donnent leurs habitudes de penser, ils en font une phrase d'enfant. G'est un phénomène auquel on pourrait donner, en le comparant à ce qui se passe pour la nutrition, le nom d'assimilation verbologique.

Nous avons dit que cette altération des mots par la mémoire se fait d'une manière assez uniforme pour les enfants, qui choisissent presque toujours le même synonyme. Pour faire comprendre ce que sont ces synonymes, il faut donner des exemples.

Nous publions ci-après une double liste : à gauche sont les mots et les groupes de mots contenus dans la dictée; à droite, les mots substitués par synonymie. Pour mieux nous faire comprendre, nous avons donné parfois une partie de la phrase; le mot ou le groupe de mots qui ont été substitués sont en italiques. Entre parenthèses nous avons indiqué le nombre de fois que les mots ont été reproduits exactement (par exemple dans la liste de gauche) et le nombre de fois qu'ils ont été remplacés. Ainsi, le premier mot de nos listes est a obtenu; ce verbe a été souvent remplacé; il a été conservé 98 fois et remplacé par a recu 38 fois. Nous n'avons pas indiqué dans notre liste sur combien d'élèves les expériences ont été faites; on trouverait au besoin ce nombre dans les tableaux précédents; il suffira de se rappeler ici, pour l'interprétation de ces résul- 


\section{A. BINET ET V. henRI. - MÉmotre des PHRases}

tats, que ce nombre est en moyenne de 35 pour chaque classe. Il faut ajouter que tous les élèves, quand le souvenir d'un mot dicté leur manque, ne le remplacent pas par le même synonyme; d'autres sont employés. Il y aurait certainement quelque intérêt, au point de vue de la psychologie du langage, à reproduire d'une manière complète la série desynonymes trouvés par les élèves; si nous ne le faisons pas, c'est tout simplement pour ne pas allonger outre mesure un article déjả considérable.

\section{MOTS DICTÉS :}

a obtenu (98 f., 4 classes). de sa mère (117 f., 4 classes). un joli ( $52 \mathbf{f} ., 4$ classes). en récompense (83 f., 4 classes). de sa bonne conduite (96 f., 4 classes).

s.amusera (124 f., 4 classes). une fois (110 f., 4 classes). terminé (127 f., 4 classes). se brise (132 f., 4 classes). le réparera ( 127 f., 4 classes).

de suite (131 f., 4 classes). plusieurs enfants (16 f.). s'amusaient (25 f.). eut $s a$ main ( $4 \mathrm{f}$.). écrasé (4 f.). d'où il a été porté (0 f.).

une vieille paysanne (13 f.). s'élanca sur elle (12 f.). et la mordit (33 f.). à plusieurs reprises (23 f.). Un commencement d'incendie (17 f.).

s'est déclaré (31 f.).

dans lappartement (8f.). par suite de l'imprudence( $19 \mathrm{f}$.). qui en tenant une bougie (6 f.). trop près des tentures (15 f.). avertis à temps (5 f.). ont pu éteindre (21 f.).

\section{SYNONYMES SUBSTITUÉS :}

a recu (38 f.).

par sa mère ( $8 \mathrm{f}$.). un beau (31 f.), petit (7 f.).

pour (20 f.), par (2 f.), à cause (2f.).

travail (15 f.), tenue ( 2 f.).

jouera (14 f.).

après (16 f.), quand (9 f.).

fini (9 f.).

se casse (3 f.). s'abime (1 f.).

le raccommodera (5 f.), rarrangera (1 f.).

tout de suite ( $2 \mathrm{f}$.$) .$

des enfants (15 f.).

jouaient (10 f.).

eut la main (24 f.).

prise (20 fr.), broyée ( $7 \mathrm{f}$.), cassée ( $2 \mathrm{f}$.$) .$ d'où il a été reconduit (14 f.), transporté $(9 \mathrm{f}$.

une vieille femme (14 f.).

sauta (8f.), se dressa (2f.), se jeta $1 \mathrm{f}$.).

la piqua (3 f.).

plusieurs fois ( $7 \mathrm{f}$.).

de feu (ö f.).

a pris (1 f.), est arrivé (1 f.).

chez (26 f.).

par (2 f.), à cause (2 f.).

chandelle (14 f.), lampe (3̈ f.).

rideaux (8 f.), tapis (3 f.), broderies (1 f.).

arrivés à temps (11 f.), venus à temps

(5 f.), parvenus à temps (3 f.).

se rendirent maitre (3 f.), ont $a r-$ rêté (1 f.) 
rapidement (0 f.).

ce commencement d'incendie (3 f.).

d'un grand prix (2 f.).

ont été dévorés par le feu (0 f.).

Un fait bien curieux vient de se passer ( $22 \mathrm{f}$.).

un vapeur (20 f.).

qui porte le nom (11 f.). en quelques instants (3 f.), aussi$t o t$ (1 f.).

le feu (13 f.), les flammes (1 f.). grande valeur (4 f.).

brûlès (17 f.), ont pris feu (2 f.), détrutits (1 f.).

vient d'arriver (7 f.).

un navire ( $8 \mathrm{f.}$ ).

du nom (10 f.), nommé (7 f.).

L'examen attentif de cette liste montre que la différence est souvent très légère entre le mot entendu et le mot remplacé ; mais si légère qu'elle soit, elle est toujours dans le sens que nous avons indiqué, c'est-à-dire dans le sens du langage enfantin. Le procédé expérimental que nous avons employé pour révéler cette différence est comparable à une balance extrêmement juste et sensible, qui marque des différences de poids très minimes.

$3^{\circ}$ L'enfant, en reproduisant une phrase ou une série de phrases, a une tendance à en simplifier la syntaxe.

Ceci est un second effet du même phénomène que nous avons appelé l'assimilation verbologique, c'est-à-dire la tendance de l'enfant à se souvenir des choses en les faisant siennes, en leur imprimant le cachet de sa personnalité. Ce qui est vrai pour les mots et les groupements de mots est également vrai pour la syntaxe; l'enfant remplace les constructions de phrases complexes par des constructions plus simples et mieux en harmonie avec son langage.

Nos expériences n'étaient nullement organisées en vue de l'étude de cette question, sur laquelle notre attention s'est portée au moment où nous avions en main les copies des élèves; nous n'avons par conséquent pas recueilli autant de résultats que la chose serait utile; nous nous contentons simplement de signaler l'intérêt de la question, en indiquant un seul exemple. Le récit suivant : - Le petit Emile a obtenu de sa mère un joli cheval mécanique en récompense de sa bonne conduite à l'école ", contient deux propositions dont la seconde est subordonnée à la première. Gette construction de phrases a été conservée par tous les élèves sans exception quand ils ont écrit la phrase aussitôt après l'avoir entendue; à cette première épreuve, ils ont fait quelques erreurs sur les mots (voir tableau I), mais 
A. BINET ET V. IIENRI. - MÉMOIRE DES PHRASES

pas d'erreur de syntaxe, ce qui semble montrer que la mémoire de syntaxe est meilleure que la mémoire des groupes de mots, quand il s'agit de retenir des phrases courtes. A la seconde épreuve, qui a lieu 20 jours après, les oublis de mots ont augmenté, et en outre des oublis de construction commencent à se manifester.

Sur 33 élèves qui ont pris part à cette expérience, 22 ont conservé la construction exacte et 11 ont modifié cette construction; sur ces derniers, il y en a 6 qui prennent la mère comme sujet de la préposition, comme dans le cas suivant :

- La mère du petit Emile lui a donné un cheval mécanique en récompense de sa bonne conduite à l'éccle. "Cette phrase ne diffère pas beaucoup par sa simplicité de la phrase dictée ; elle est, on peut le dire, équivalente à la première; mais on remarque une simplification considérable chez les autres 5 élèves qui ont divisé la phrase primitive en deux parties comme dans l'exemple suivant :

- Le petit Émile a été sage à l'école, pour le récompenser sa mère lui a donné un cheval mécanique ..

Nous n'insistons pas davantage et répétons que la question mérite d'être reprise dans des expériences méthodiques, où différentes complexités de syntaxe seront offertes à la mémoire des élèves.

\section{$\mathbf{V}$}

\section{ERREURS PAR IMAGINATION}

Dans toutes les expériences qui portent sur une phrase de plus de 20 mots la mémoire produit, chez plus de la moitié des élèves, une altération légère,presque insensible, du sens de la phrase.

Nous constatons ici un fait qui paraît avoir une importance primordiale; chacun sait, par expérience personnelle, que bien souvent on croit avoir lu tel passage dans un livre, entendu tel mot dans la conversation; et quand on peut faire le contrôle de son souvenir, on s'aperçoit qu'on s'est gravement trompé. La mémoire, dépositaire infidèle, a modifié, altéré les perceptions qu'on lui a confiées.

Dans nos expériences collectives, nous pouvons étudier de très près ces altérations du sens des phrases par la mémoire. Disons 
d'abord que le nombre de ces substitutions est plus faible que celui des synonymes. C'est ce que montre le tableau II et aussi le graphique 2 , où l'on verra également que le nombre de substitutions varie beaucoup avec la nature des phrases.

En outre, les substitutions par analogies de sens sont personnelles à chaque enfant; il est rare que plusieurs enfants tombent sur le même mot, comme cela avait lieu pour les synonymes; ceci nous démontre qu'en ce qui concerne le vocabulaire les enfants sont bien plus uniformes que pour les phénomènes intimes de l'idéation.

En cherchant à mettre un peu d'ordre dans ces substitutions qui présentent des variétés très nombreuses, nous distinguerons les espèces suivantes :

1. A un nom on substitue un autre nom qui lui ressemble plus ou moins; à un nombre quelconque on substitue un nombre différent. Exemples (dans les listes qui suivent, le mot dicté est à gauche).

Victor Antiquet (14).

La veuve Mouillet (19).

âgée de 64 ans (21).

53 , rue Jacob (30).
Ifenri Antiquet (4), Riquelet (1), Tiquet (1), Louis (1).

La jeune (1) La vieille (2) Mine (1) La veuve Nicolet (1) La nommée Jeanne. 80 ans (1) 63 ans (1) 70 ans (1) 34 ans (1) 84 ans (1).

2, rue Jacob (1) 12, rue Jacob (1)

Dans ces cas, parfois l'enfant garde un souvenir du son, et il trouve un nom qui se rapproche de celui qu'on lui a donné; parfois il ne se rappelle qu'une chose, c'est qu'un nom a été donné, et comme il ne le connait plus, il en invente un qui n'a aucun rapport avec celui qu'on a dicté. Ainsi Louis pour Victor Antiquet, la nommée Jeanne au lieu de la veuve Mouillet, etc. Il en est de même pour les chiffres. L'enfant se rappelle qu'un chiffre a été dit, mais il ne se rappelle plus lequel.

$2^{\circ}$ Un objet est remplacé par un objet analogue, pouvant jouer dans la phrase le même rôle.

$3^{\circ}$ L'altération de mémoire ne consiste pas dans un changement, mais dans une addition; c'est l'imagination qui travaille, sans doute à l'insu de l'enfant, et qui ajoute des détails de son cru. Nous aurions ici un grand nombre d'exemples à citer, montrant le travail de l'imagination à tous ses degrés; au degré le plus inférieur, c'est à peine une nuance : c'est la précision apportée dans un fait qui, dans le texte originál, est resté vague; par 
exemple, l'enfant a entendu : Dimanche, et il écrit : Dimanche dernier ou Dimanche soir; on lui a parlé de plusieurs enfants, il écrit : plusieurs jeunes enfants. De telles modifications passeraient inapercues si on n'y faisait grande attention. - En voici quelques exemples:

Nous avons réuni ici les substitutions des genres 2 et 3 , parce qu'il est assez difficile de les distinguer.

Le petit Emile a obtenu cheval mécanique

son ami

jouera avec lui

le jeudi et le dimanche si le cheval...

Dimanche

plusieurs enfants

jouaient à faire marcher

une machine à mortier

l'un d'eux

eut sa main

écrasée

il a recu les premiers soins dans une pharmacie

d'où il a èté porté chez ses parents.

Un commencement d'incendie (17)

s'est déclaré hier (26)

en tenant une bougie

l'a approchce trop près des tentures

rapidement.

Une vieille paysanne (13)

habitant une petite maison sur la route déserte (4)

avait conduit (3)

son troupeau (21)
On lui a acheté (1) on lui a promis (1) cheval de bois (3) cheval de bois blane (1) son petit camarade (5) Emile (1)

jouera avec le cheval (5)

tous les jeudis (1) le matin du jeudi ( $t$ ) si la mécanique (1).

Dimanche dernier (1) L'autre jour (3)

Hier (1) Samedi (1)

plusieurs petits enfants (1)

à faire tourner (1) à faire rouler (1)

la mécanique (1) une voiture (1) une brouette (1) une écrase-machine (1)

le plus jeune (1)

eut le bras (1) eut la jambe (1)

emportée (1)

dans une pharmacie voisine (4) chez un médecin (1)

à son domicile (1) chez lui (1) chez sa mère.

Un violent incendie (1)

hier soir (3) samedi (1)

une lampe

l'avait posée (1) laissé tomber (1) mis sur la table (1) pendant son absence (1) en un quart d'heure (1) aussitôt (1) après de grands efforts (1).

Une pauvre vieille (2) Une vieille femme (1) Une pauvre femme (1) Une femme arabe (1)

une grande maison (1) une vieille maison (1)

dans la rue déserte (2) dans un village (1) dans la campagne (1)

allait tous les jours avec son troupeau (1) son pauvre petit troupeau (1) ses moutons (10) 
dans les champs (10̈)

pendant qu'elle faisait de l'herbe pour ses animaux (9) une vipère cachée derrière

les fagots

la mordit au poignet. dans la campagne (1) dans les prés (4) sur la route (3) dans une forêt déserte (1)

pour ses lapins (1) pour ses enfants (1) pendant qu'elle faisait des fagots (1) cachée dans l'herbe (2) dans les buissons (2) dans un arbre (1) dans le tronc d'un arbre (1) dans de vieux fagots (1)

à la main (1).

En étudiant de près ces additions faites par l'imagination à la mémoire, on s'aperçoit qu'elles sont parfois faciles à expliquer : elles proviennent de l'ensemble des idées contenues dans la phrase; ces idées ont suggéré à l'enfant des images qui leur sont habituellement associées; pensant à une vieille femme qui conduit un troupeau; il a imaginé un troupeau de moutons; ou bien voyant la femme accroupie et faisant de l'herbe, il a pensé à ses lapins. Dans d'autres cas, l'explication est moins facile.

$4^{\circ}$ Comme dernière altération de la mémoire nous signalerons l'altération émotionnelle : dans certaines copies, rares à la vérité, nous notons que l'enfant a exagéré le sentiment exprimé par le texte dicté; ainsi le serpent devient le dangereux, l'affreux serpent; la vipère la mordit cruellement, elc. Il est probable que, dans des récits plus émouvants que les nôtres, ce caractère émotionnel aurait pris un plus grand développement.

En quelques mots très brefs nous conclurons cette étude, qui a été une sorte de voyage d'exploration dans un domaine jusqu'ici presque entièrement inconnu. Nous avons vu que : $1^{\circ}$ la mémoire des phrases (ou des idées) présente un accroissement faible, mais constant, avec l'âge ; $2^{\circ}$ elle est, dans certaines conditions que nous avons fixées, vingt-cinq fois supérieure $\mathfrak{a}$ la mémoire des mots isolés; $3^{\circ}$ le nombre des oublis augmente rapidement avec la longueur des phrases el des séries de phrases; pour une phrase de 20 mots ( 8 groupes de mots) il a été de $\frac{1}{80}$; pour une série de phrases de 80 mots (24 groupes de mots), il a été de $\frac{1}{3} \cdot 4^{\circ}$ Les pertes de mémoire portent sur les parties 1 accessoires du récit, et non sur les parties essentielles, qui se trouvent ainsi comme disséquées ; par parties essentielles, il faut entendre celles qui ont une importance psychologique et aussi celles qui ont une importance logique $5^{\circ}$ Les substitu- 
A. BINET ET V. HENRI. - MÉMOIRE DES - PHRASES

tions de synonymes sont plus nombreuses que les oublis térmplets pour les textes courts; c'est le contraire pour des textes longs. $6^{\circ}$ Les enfants ont une tendance à simplifier la syntaxe et à remplacer les mots dictés par des synonymes du langage familier. G'est ce qu'on peut appeler l'assimilation verbologique. $7^{\circ}$ Les enfants, en reproduisant de mémoire des phrases un peu longues, ont une tendance fréquente à altérer d'une manière très légère le sens des phrases; cette altération est de deux genres différents : intellectıelle et émotionnelle.

Alfred Binet ét Victor Henri. 